\title{
New Realization of Periodic Cycled Separation
}

Bjarne Toftegård $^{\mathrm{a}, \mathrm{b}}$, Charlotte H. Clausen ${ }^{\mathrm{a}, \mathrm{c}}$, Sten B. Jørgensen ${ }^{\mathrm{a}}$, Jens Abildskov ${ }^{\mathrm{a} *}$

${ }^{a}$ CAPEC-PROCESS, Department of Chemical and Biochemical Engineering, Technical University of Denmark. Building 229, DK-2800 Kgs. Lyngby, Denmark

${ }^{\mathrm{b}}$ Present address: Havneholmen 80, 4.tv., 1561 København V, Denmark

${ }^{\mathrm{c}}$ Present address: Novo Nordisk A/S, Hagedornsvej 1, 2820 Gentofte, Denmark

* Corresponding author e-mail: ja@kt.dtu.dk

\section{Supporting Information}


Table S1. Tray efficiency results. Periodic Trays.

\begin{tabular}{cccccc}
\hline$H$ (moles) & $G$ (moles/s) & $m$ & Slope $d p H / d t$ & $\varepsilon$ & $H / G(\mathrm{~s})$ \\
\hline 795.4 & 10.3 & 0.68 & -0.0059 & 3.0 & 77.2 \\
772.2 & 10.5 & 0.75 & -0.0057 & 2.6 & 73.9 \\
494.3 & 10.6 & 0.78 & -0.0080 & 2.2 & 46.9 \\
581.3 & 10.6 & 0.79 & -0.0075 & 2.4 & 55.0 \\
630.0 & 9.7 & 0.72 & -0.0068 & 2.9 & 65.1 \\
481.1 & 8.4 & 0.73 & -0.0066 & 2.4 & 57.1 \\
500.6 & 8.4 & 0.72 & -0.0072 & 2.8 & 59.6 \\
161.8 & 10.3 & 0.80 & -0.0139 & 1.3 & 15.7 \\
206.4 & 10.5 & 0.80 & -0.0126 & 1.4 & 19.7 \\
266.9 & 10.5 & 0.80 & -0.0104 & 1.5 & 25.5 \\
140.9 & 10.7 & 0.80 & -0.0137 & 1.0 & 13.1 \\
137.3 & 10.8 & 0.79 & -0.0137 & 1.0 & 12.8 \\
\hline & & & & & \\
\hline
\end{tabular}


Table S2. Periodic Column Separations

\begin{tabular}{lrrrrrrrrrrrr}
\hline Experiment & $T\left({ }^{\circ} \mathrm{C}\right)$ & $T P(\mathrm{~s})$ & $D P\left(\mathrm{~mm} \mathrm{H}_{2} \mathrm{O}\right)$ & $L(\mathrm{~mol} / \mathrm{s})$ & $G(\mathrm{~mol} / \mathrm{s})$ & $m$ & $x_{f}$ & $x_{p}$ & $S$ & $N_{i d}$ & $F P$ & $C E_{p}$ \\
\hline PE1 & 24.1 & 120 & 60 & 2.9 & 8.4 & 0.91 & 0.0005090 & 0.0000216 & 23.6 & 2.8 & 0.00948 & 0.94 \\
PE2 & 22.8 & 80 & 60 & 4.4 & 8.4 & 0.86 & 0.0005090 & 0.0000374 & 13.6 & 3.6 & 0.01424 & 1.21 \\
PE3 & 23.5 & 40 & 60 & 8.8 & 8.3 & 0.88 & 0.0005090 & 0.0001700 & 3.0 & 2.8 & 0.02878 & 0.93 \\
PE4 & 25.2 & 140 & 60 & 2.6 & 8.3 & 0.95 & 0.0005090 & 0.0000213 & 23.9 & 2.5 & 0.00833 & 0.83 \\
PE5 & 25.1 & 100 & 60 & 3.7 & 8.3 & 0.94 & 0.0005090 & 0.0000258 & 19.7 & 3.2 & 0.01218 & 1.07 \\
PE6 & 25.0 & 160 & 60 & 2.1 & 8.4 & 0.94 & 0.0005090 & 0.0000189 & 26.9 & 2.3 & 0.00692 & 0.76 \\
PE7 & 25.8 & 60 & 60 & 5.9 & 8.4 & 0.97 & 0.0006220 & 0.0000805 & 7.7 & 3.2 & 0.01911 \\
PE8 & 24.5 & 200 & 60 & 1.8 & 8.3 & 0.92 & 0.0006220 & 0.0000179 & 34.8 & 2.3 & 0.00599 & 1.08 \\
PE9 & 24.6 & 70 & 60 & 5.3 & 8.3 & 0.92 & 0.0006220 & 0.0000647 & 9.6 & 3.5 & 0.01716 & 1.15 \\
PE10 & 23.6 & 100 & 60 & 4.4 & 6.9 & 0.89 & 0.0006220 & 0.0000719 & 8.7 & 3.5 & 0.01742 \\
PE11 & 24.5 & 100 & 50 & 4.3 & 6.9 & 0.92 & 0.0006220 & 0.0000628 & 9.9 & 3.4 & 0.01678 & 1.17 \\
PE12 & 24.4 & 100 & 40 & 2.4 & 6.9 & 0.92 & 0.0006220 & 0.0000220 & 28.3 & 3.0 & 0.00941 & 0.99 \\
PE13 & 20.1 & 100 & 48 & 3.5 & 7.0 & 0.76 & 0.0006300 & 0.0000617 & 10.2 & 3.4 & 0.01361 \\
PE14 & 20.6 & 70 & 48 & 4.8 & 7.0 & 0.78 & 0.0006300 & 0.0001120 & 5.6 & 3.5 & 0.01874 \\
PE15 & 21.4 & 55 & 48 & 5.3 & 7.0 & 0.81 & 0.0006300 & 0.0001380 & 4.6 & 3.1 & 0.02054 & 1.14 \\
PE16 & 22.5 & 85 & 48 & 3.7 & 7.0 & 0.85 & 0.0006300 & 0.0000534 & 11.8 & 3.4 & 0.01429 & 1.04 \\
PE17 & 24.5 & 47 & 48 & 5.9 & 7.0 & 0.92 & 0.0006300 & 0.0001480 & 4.3 & 2.8 & 0.02316 & 0.94 \\
PE18 & 23.9 & 70 & 80 & 7.4 & 8.3 & 0.90 & 0.0005940 & 0.0001310 & 4.5 & 3.5 & 0.02410 \\
PE19 & 24.3 & 70 & 40 & 2.9 & 8.4 & 0.91 & 0.0005940 & 0.0000191 & 31.1 & 3.0 & 0.00931 \\
PE20 & 24.3 & 70 & 50 & 4.2 & 8.3 & 0.92 & 0.0005940 & 0.0000387 & 15.4 & 3.3 & 0.01359 & 1.15 \\
\hline
\end{tabular}


Table S3. Sieve trays Column Separations.

\begin{tabular}{|c|c|c|c|c|c|c|c|c|c|c|}
\hline Experiment & $T\left({ }^{\circ} \mathrm{C}\right)$ & $L(\mathrm{~mol} / \mathrm{s})$ & $G(\mathrm{~mol} / \mathrm{s})$ & $m$ & $x_{f}$ & $x_{p}$ & $S$ & $N_{i d}$ & $F P$ & $C E_{c}$ \\
\hline $\mathrm{C} 1$ & 22.5 & 12.2 & 8.2 & 0.84 & 0.000682 & 0.000386 & 1.8 & 1.5 & 0.040 & 0.51 \\
\hline $\mathrm{C} 2$ & 22.5 & 7.2 & 8.2 & 0.84 & 0.000682 & 0.000265 & 2.6 & 1.7 & 0.024 & 0.56 \\
\hline C3 & 22.7 & 21.4 & 8.2 & 0.85 & 0.000682 & 0.000490 & 1.4 & 1.5 & 0.071 & 0.50 \\
\hline $\mathrm{C} 4$ & 24.5 & 4.4 & 8.2 & 0.92 & 0.000495 & 0.000132 & 3.8 & 1.4 & 0.015 & 0.47 \\
\hline $\mathrm{C} 5$ & 25.2 & 3.4 & 8.2 & 0.95 & 0.000495 & 0.000091 & 5.5 & 1.5 & 0.011 & 0.50 \\
\hline $\mathrm{C} 6$ & 25.5 & 2.3 & 8.1 & 0.96 & 0.000495 & 0.000056 & 8.9 & 1.5 & 0.008 & 0.52 \\
\hline $\mathrm{C7}$ & 25.5 & 2.6 & 6.2 & 0.96 & 0.000495 & 0.000075 & 6.6 & 1.7 & 0.011 & 0.57 \\
\hline $\mathrm{C} 8$ & 25.1 & 17.6 & 8.2 & 0.95 & 0.000614 & 0.000406 & 1.5 & 1.3 & 0.058 & 0.43 \\
\hline C9 & 24.7 & 27.1 & 8.2 & 0.93 & 0.000614 & 0.000471 & 1.3 & 1.2 & 0.090 & 0.39 \\
\hline $\mathrm{C} 10$ & 25.0 & 17.3 & 6.2 & 0.94 & 0.000614 & 0.000442 & 1.4 & 1.3 & 0.076 & 0.44 \\
\hline C11 & 24.9 & 8.7 & 6.2 & 0.94 & 0.000614 & 0.000332 & 1.8 & 1.4 & 0.038 & 0.45 \\
\hline $\mathrm{C} 12$ & 24.6 & 4.8 & 6.2 & 0.93 & 0.000614 & 0.000226 & 2.7 & 1.4 & 0.021 & 0.46 \\
\hline $\mathrm{C} 13$ & 24.7 & 2.6 & 6.2 & 0.93 & 0.000614 & 0.000090 & 6.8 & 1.8 & 0.011 & 0.59 \\
\hline
\end{tabular}


Table S4. Sulzer BX Gauze Packing Column Separations.

\begin{tabular}{lrrrrrrrrr}
\hline Experiment & $T\left({ }^{\circ} \mathrm{C}\right)$ & $L(\mathrm{~mol} / \mathrm{s})$ & $G(\mathrm{~mol} / \mathrm{s})$ & $m$ & $x_{f}$ & $x_{p}$ & $S$ & $N_{i d}$ & $F P$ \\
\hline P1 & 22.8 & 5.9 & 9.9 & 0.85 & 0.000542 & 0.000181 & 3.0 & 1.3 & 0.01633 \\
P2 & 22.9 & 9.2 & 9.8 & 0.86 & 0.000542 & 0.000269 & 2.0 & 1.1 & 0.02541 \\
P3 & 23.5 & 16.1 & 9.8 & 0.88 & 0.000542 & 0.000341 & 1.6 & 1.1 & 0.04437 \\
P4 & 24.0 & 4.4 & 6.3 & 0.90 & 0.000542 & 0.000161 & 3.4 & 1.7 & 0.01888 \\
P5 & 24.8 & 8.5 & 6.3 & 0.94 & 0.000542 & 0.000317 & 1.7 & 1.0 & 0.03644 \\
P6 & 25.0 & 12.3 & 6.3 & 0.94 & 0.000542 & 0.000364 & 1.5 & 1.0 & 0.05268 \\
P7 & 26.1 & 15.6 & 6.3 & 0.99 & 0.000542 & 0.000389 & 1.4 & 1.0 & 0.06731 \\
P8 & 26.3 & 26.2 & 6.3 & 0.99 & 0.000542 & 0.000442 & 1.2 & 0.9 & 0.11275 \\
P9 & 20.8 & 1.9 & 6.3 & 0.78 & 0.000573 & 0.000121 & 4.7 & 1.2 & 0.00813 \\
P10 & 21.5 & 1.8 & 6.3 & 0.81 & 0.000573 & 0.000122 & 4.7 & 1.2 & 0.00789 \\
P11 & 22.1 & 3.5 & 6.3 & 0.83 & 0.000573 & 0.000163 & 3.5 & 1.5 & 0.01497 \\
P12 & 23.4 & 4.6 & 6.3 & 0.88 & 0.000573 & 0.000198 & 2.9 & 1.5 & 0.01975 \\
P13 & 23.0 & 3.3 & 6.3 & 0.87 & 0.000573 & 0.000144 & 4.0 & 1.5 & 0.01421 \\
P14 & 22.9 & 2.9 & 9.8 & 0.86 & 0.000573 & 0.000082 & 7.0 & 1.5 & 0.00819 \\
P15 & 23.0 & 2.4 & 9.7 & 0.86 & 0.000573 & 0.000061 & 9.5 & 1.6 & 0.00682 \\
P16 & 23.3 & 1.9 & 9.7 & 0.87 & 0.000573 & 0.000038 & 15.0 & 1.7 & 0.00534 \\
\hline
\end{tabular}

